\title{
Distribution of Chitinolytic Enzymes in the Organs and cDNA Cloning of Chitinase Isozymes from the Liver of Golden Cuttlefish Sepia esculenta
}

\author{
Ryo Nishino, Hiromi Kakizaki, Hideto Fukushima, Masahiro Matsumiya \\ Department of Marine Science and Resources, College of Bioresource Sciences, Nihon University, \\ Fujisawa, Kanagawa, Japan \\ Email: matsumiya@brs.nihon-u.ac.jp
}

How to cite this paper: Nishino, R., Kakizaki, H., Fukushima, H. and Matsumiya, M. (2017) Distribution of Chitinolytic Enzymes in the Organs and cDNA Cloning of Chitinase Isozymes from the Liver of Golden Cuttlefish Sepia esculenta. Advances in Bioscience and Biotechnology, 8, 361-377. https://doi.org/10.4236/abb.2017.810026

Received: September 7, 2017

Accepted: October 10, 2017

Published: October 13, 2017

Copyright $\odot 2017$ by authors and Scientific Research Publishing Inc. This work is licensed under the Creative Commons Attribution International License (CC BY 4.0).

http://creativecommons.org/licenses/by/4.0/

(c) (i) Open Access

\begin{abstract}
The distribution of chitinolytic enzymes in eight organs of the golden cuttlefish Sepia esculenta was determined. Chitinase activity (activity of endo-type chitinolytic enzyme) was measured using $p \mathrm{NP}-(\mathrm{GlcNAc})_{\mathrm{n}}(\mathrm{n}=2,3)$ as substrates, with high activity detected in the liver, posterior salivary gland, and stomach. $\beta$ - $N$-acetylhexosaminidase (Hex) activity (activity of exo-type chitinolytic enzyme) was determined using $p \mathrm{NP}-(\mathrm{GlcNAc})$ as a substrate, and high activity was observed in six organs, including the liver, branchial heart, posterior salivary gland, and stomach. In addition, two chitin-binding proteins (CBP-A, CBP-B) were isolated from the liver using a chitin affinity column. Two full-length cDNAs (SeChi-1: 1484 bp; SeChi-2: 1748 bp) encoding chitinases were obtained from the liver of $S$. esculenta. SeChi-1 contained a 1377-bp open reading frame (ORF) encoding 459 amino acids, and SeChi-2 contained a 1656-bp ORF encoding 552 amino acids. Domain structures predicted from the deduced amino acid sequences of SeChi-1 and SeChi-2 (SeChi-1, SeChi-2) contained signal peptides, a GH Family 18 catalytic domain, one chitin binding domain (CBD) in SeChi-1, and two CBDs in SeChi-2. Proteome analysis revealed that 125 peptide residues of CBP-A were present in SeChi-1, and 116 peptide residues of CBP-B were present in SeChi-2. Organ expression analysis revealed that SeChi-1 and SeChi-2 were expressed only in the liver of $S$. esculenta. Phylogenetic analysis of SeChi-1, SeChi-2, and $\mathrm{GH}$ family 18 chitinases revealed that SeChi-2 belongs to a group of previously reported squid chitinases, while SeChi-1 does not belong to any previously reported group of mollusk chitinases.
\end{abstract}




\section{Keywords}

Chitinolytic Enzyme, Chitinase, Distribution, cDNA Cloning, Golden Cuttlefish Sepia esculenta

\section{Introduction}

Chitin, a $\beta$-1,4-linked polysaccharide of $N$-acetyl-D-glucosamine (GlcNAc), is an abundant reproducible biomass found widely in the exoskeletons of arthropods, the cell walls of fungi, and the cuticles of nematodes [1] [2] [3] [4]. Chitinolytic enzymes can be classified into two categories according to their degradation patterns: endo-type chitinolytic enzymes, called chitinase (EC 3.2.1.14), which degrade a random chitin polymer to produce chitin oligosaccharides (GlcNAc) $)_{\mathrm{n}}$, and exo-type chitinolytic enzymes, called $\beta$ - $N$-acetylhexosaminidase (Hex) (EC 3.2.1.52), which degrade (GlcNAc) ${ }_{n}$ from the nonreducing end of it to produce GlcNAc [5] [6]. Chitinases are found in various living organisms, including animals, plants, and microorganisms, and have important roles in biological processes, such as digestion, morphological changes during growth, and immunity [5] [7]. Chitinases are classified into glycoside hydrolase (GH) family 18 or 19, based on the homology of amino acid sequences [4] [8] and catalytic mechanisms in their active domains [9] [10]. GH family 18 chitinases are found widely in biology, including in microorganisms, animals, and plants [5]. Conversely, GH family 19 chitinases are found mainly in plants [11].

In marine animals, studies have reported the purification, properties, and cDNA cloning of chitinase isozymes mainly obtained from the fish stomach, which are involved in digestion [12]-[17]. Chitinases in the fish stomach are classified into two groups based on differences in their primary structure and the patterns of degradation of (GlcNAc) ${ }_{\mathrm{n}}$ : acidic fish chitinase-1 (AFCase-1) and acidic fish chitinase-2 (AFCase-2) [16] [17] [18] [19]. Conversely, studies reporting the cDNA cloning and expression of chitinases and chitinase-like proteins from bivalves and gastropods, which are mollusks, have noted that these play roles in shell formation [20] [21] [22], immunity [23] [24] [25] [26], and digestion [27]. Chitinase isozymes have been purified and studied from the liver of Decembrachiata (squid and cuttlefish), and are involved in digestion [28] [29] [30] [31]. Furthermore, two chitinase isozymes have been reported in the liver of Japanese common squid, and identified based on differences in molecular weight and N-terminal amino acid sequences [29] [30], and two chitinase isozymes have been reported in the liver of spear squid, and identified based on expressed sequence tag (EST) analysis [32]. However, the full-length genes have not yet been determined. Conversely, chitinases have been obtained from the posterior salivary gland of octopus [33] and cuttlefish [34], and found to act as poison. A chitotriosidase gene, which is involved in the induction of luminescent bacteria, has been found in the light organ of the Hawaiian bobtail squid Euprym- 
nascolopes [35]. Thus, the roles of mollusk chitinases are not limited to digestion and range widely; thus, many isozymes exist to support these different roles.

Golden cuttlefish Sepia esculenta, used in the present study, belongs to Decembrachiata and is a type of mollusk that mainly ingests crustaceans, which contain chitinous substances, and fish [36]. We have previously reported the distribution of chitinase activity using glycolchitin as the substrate [37] and purification and properties of a chitinase obtained from the liver of $S$. esculenta [31]; however, no findings of enzyme proteins and genes corresponding with the chitinase isozymes have been reported. In this study, we first observed the distribution of chitinase activity using two kinds of chitinase specific substrates in the body of $S$. esculenta, and isolated two types of chitin-binding proteins (CBPs) from the liver that exhibited particularly high chitinase activity. Next, we cloned chitinase genes from the liver and obtained two types of full-length genes. Furthermore, the organ expression of the genes was analyzed, domain structures were compared, and phylogenetic analyses was performed based on the deduced amino acid sequences. The relationship between the two types of CBPs was elucidated and the different chitinase genes obtained were examined by proteome analysis. In summary, this study is the first to discuss the distribution of chitinolytic enzymes in $S$. esculenta, the presence of chitinase isozymes and features of their domain structure, and the positioning of chitinase isozymes in phylogenetic analysis.

\section{Materials and Methods}

\subsection{Materials}

Fresh S. esculenta was purchased from Tsukiji Fish Market (body weight: 183 g, liver weight: $9.5 \mathrm{~g})$.

\subsection{Measurement of Chitinolytic Enzyme Activity}

Organs were removed from $S$. esculenta for subsequent analysis. Each organ was homogenized in five volumes of $20 \mathrm{mM}$ phosphate buffer ( $\mathrm{pH}$ 7.2), and then the homogenate was centrifuged at $7000 \times g$ for $20 \mathrm{~min}$. The supernatant was used as the crude enzyme solution. Chitinase and Hex activities were measured using $p$-nitrophenyl $(\mathrm{GlcNAc})_{\mathrm{n}},\left(p \mathrm{NP}-(\mathrm{GlcNAc})_{\mathrm{n}}\right)(\mathrm{n}=2,3)$ (Seikagaku, Tokyo, Japan) and $p$ NP-GlcNAc (Seikagaku) as substrates, respectively, according to the method described by Ohtakara [38], with slight modification. Briefly, 3.0 $\mu \mathrm{L}$ of crude enzyme solution and $2.5 \mu \mathrm{L}$ of $4 \mathrm{mM}$ substrate solution were added to 10 $\mu \mathrm{L}$ of $0.2 \mathrm{M}$ phosphate- $0.1 \mathrm{M}$ citrate buffer ( $\mathrm{pH} 6.0$ ), and then the solution was incubated at $37^{\circ} \mathrm{C}$ for $20 \mathrm{~min}$. After incubation, $65 \mu \mathrm{L}$ of $0.2 \mathrm{M}$ sodium carbonate solution was added, and the absorbance of released p-nitrophenol was measured at $420 \mathrm{~nm}$. One-unit of chitinolytic enzyme activity $(\mathrm{U})$ was defined as the amount of enzyme that liberated $1 \mu \mathrm{mol}$ of $p$-nitrophenol per minute, and was expressed as the activity per gram of organ. 


\subsection{Isolation of CBPs from the Liver of $S$. esculenta}

Unless otherwise noted, all processes were carried out at $0^{\circ} \mathrm{C}-4^{\circ} \mathrm{C}$. Livers were collected from fresh $S$. esculenta and kept at $-80^{\circ} \mathrm{C}$ until use. The livers were homogenized with five volumes of $50 \mathrm{mM}$ sodium acetate buffer ( $\mathrm{pH} 5.5$ ) and centrifuged at $7000 \times g$ for $20 \mathrm{~min}$. Ammonium sulfate was added to the supernatant to give $70 \%$ saturation, and the preparation was left to stand for $24 \mathrm{~h}$. The precipitate was then collected by centrifuging at $7000 \times g$ for $20 \mathrm{~min}$, and dialyzed in $20 \mathrm{mM}$ sodium phosphate buffer ( $\mathrm{pH}$ 7.2). The dialyzed solution was centrifuged at $7000 \times g$ for $20 \mathrm{~min}$ and $\mathrm{NaCl}$ was added to bring the concentration to $1 \mathrm{M}$. This solution was applied to a chitin affinity column (Chitin EX column) (Funakoshi, Tokyo, Japan) $(1.5 \times 10 \mathrm{~cm})$ previously equilibrated with $20 \mathrm{mM}$ sodium phosphate buffer $(\mathrm{pH} 7.2)$ containing $1 \mathrm{M} \mathrm{NaCl}$, and the non-adsorbed fractions were eluted with the same buffer. Adsorbed fractions were eluted with $0.1 \mathrm{M}$ acetic acid. Finally, the adsorbed fractions were dialyzed with distilled water. Chitinase activity was measured using $p \mathrm{NP}-(\mathrm{GlcNAc})_{2}$.

\subsection{Amino Acid Sequence of the CBPs Isolated from the Liver of $S$, esculenta}

The chitinase-active fraction was subjected to sodium dodecyl sulfate-polyacrylamide gel electrophoresis (SDS-PAGE) and stained with AE-1360 Ez Stain Silver (ATTO, Tokyo, Japan). A gel slice was cut into small pieces and destained with destaining solution $\left(15 \mathrm{mMK}_{3}\left[\mathrm{Fe}(\mathrm{CN})_{6}\right], 50 \mathrm{mM} \mathrm{Na} 2 \mathrm{~S}_{2} \mathrm{O}_{3}\right)$. Destained gel pieces were trypsinized as described in the manual for the In-Gel Tryptic Digestion Kit (Thermo Scientific, Waltham, MA). The peptide mixtures obtained were subjected to a nanoscale liquid chromatography-electrospray (Thermo Scientific) equipped with a captive spray ionization source (Michrom Bioresources, Auburn, CA) and an Advance UHPLC System (Michrom Bioresources).

\section{5. cDNA Cloning of Chitinases from the Liver of S, esculenta}

Total RNA was extracted from the $\mathcal{S}$. esculenta liver using ISOGEN II (Nippon Gene, Tokyo, Japan) according to the manufacturer's instructions. Next, cDNA was synthesized using $1.0 \mu \mathrm{g}$ of total RNA, a PrimeScript reverse transcriptase (Takara Bio, Shiga, Japan), and an oligo dT primer (Table 1). The reaction conditions were $90^{\circ} \mathrm{C}$ for $3 \mathrm{~min}, 42^{\circ} \mathrm{C}$ for $60 \mathrm{~min}$, and $70^{\circ} \mathrm{C}$ for $10 \mathrm{~min}$. The primers used are listed in Table 1, and the primer combinations are shown in Figure 1. Internal sequences were amplified in a solution containing the synthesized cDNA, Go Taq Green Master Mix (Promega, Madison, USA), and degenerate primers designed using the conserved amino acid sequences of GH family 18 chitinases from several organisms. PCR parameters for the first PCR were as follow: initial denaturation at $95^{\circ} \mathrm{C}$ for $2 \mathrm{~min}$, followed by 35 cycles of $95^{\circ} \mathrm{C}$ for $30 \mathrm{~s}, 55^{\circ} \mathrm{C}$ for $1 \mathrm{~min}$, and $72^{\circ} \mathrm{C}$ for $2 \mathrm{~min}$. Nested PCR was performed using the same PCR parameters except that the sample was 10-fold diluted for the first PCR products. Forward and reverse primers were designed from the chitinase 


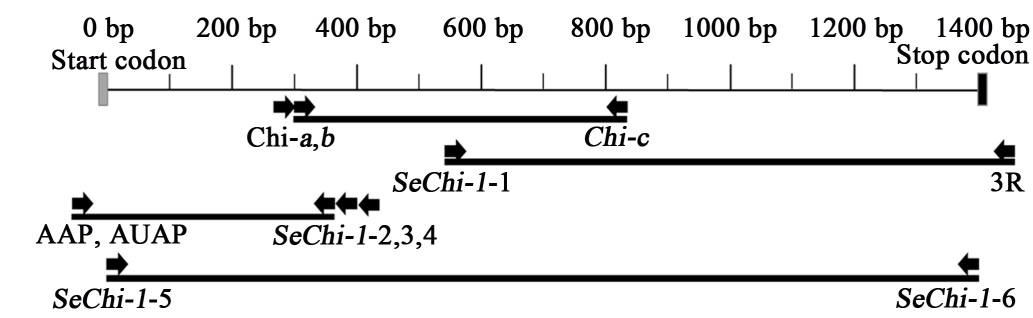

(a)

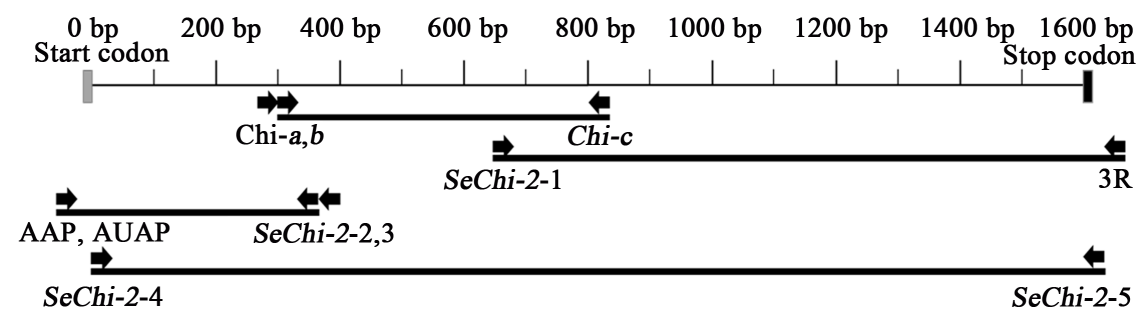

(b)

Figure 1. Schematic representation of the cDNA structure of (a) SeChi-1, (b) SeChi-2 and location of the primers. Arrowheads indicate the primers, and lines between the arrowheads indicate the amplified cDNA fragments.

Table 1. Primers used for PCR, RACE, and organ expression.

\begin{tabular}{|c|c|c|}
\hline Primer & Sequence $\left(5^{\prime}-3^{\prime}\right)$ & Purpose \\
\hline Oligo dT & CTGTGAATGCGACTACGATTTTTTTTTTTTTTTTTTT & cDNA synthesis \\
\hline$C h i-a^{*}$ & GGNGGNTGGAAYATGGG & Primary PCR \\
\hline$C h i-b^{*}$ & TNGCNGCNTTYGARTGGAAYGA & Primary PCR \\
\hline Chi-c $c^{*}$ & ANCANRANCCNTTRGTYACCCA & Primary PCR \\
\hline SeChi-1-1 & GCACCAAAGAAAAAAGTTGAT & 3'RACE \\
\hline SeChi-2-1 & GGACATAACAGCCCTCTG & 3'RACE \\
\hline $3 R$ & CTGTGAATGCGACTACGAT & 3'RACE \\
\hline SeChi-1-2 & GCCCATGTTCCAGCCACC & 5'RACE \\
\hline SeChi-1-3 & TTCGTCGTTCCACTCAAAAGC & 5'RACE \\
\hline SeChi-1-4 & CCCATTCAGTTTGGCAAAAGC & 5'RACE \\
\hline SeChi-2-2 & CGCTACCATGGCAGTGAAAGG & 5'RACE \\
\hline SeChi-2-3 & CTTCATCCATGGTTCAGATTC & 5'RACE \\
\hline AAP & GGCCACGCGTCGACTAGTACGGGIIGGGIIGGGIIG & 5'RACE \\
\hline AUAP & GGCCACGCGTCGACTAGTAC & 5'RACE \\
\hline SeChi-1-5 & ACACATTACAGCAAA & Full-length ORF \\
\hline SeChi-1-6 & TAATAAATACCAAGATTAT & Full-length ORF \\
\hline SeChi-2-4 & CGAGTTCTGGTGGACAAA & Full-length ORF \\
\hline SeChi-2-5 & GGCTGAAAAATAAAATGT & Full-length ORF \\
\hline$\beta$-actin-a & GGTATGTGCAAAGCTGGTTTT & Organ expression \\
\hline$\beta$-actin-b & GTGGGTGACACCATCACCAGA & Organ expression \\
\hline SeChi-1-a & GAAACTTTGATGGTTTGGACAT & Organ expression \\
\hline SeChi-1-b & TGTGTCCATACAAAGCAATTCC & Organ expression \\
\hline SeChi-2-a & GAGAAATACCCACTGCTGAAGA & Organ expression \\
\hline SeChi-2-b & AGAACAGTTAGGAATAGCGGAT & Organ expression \\
\hline
\end{tabular}

*Degenerate primers; 5'AAP: 5'RACE abridged anchor primer; AUAP: abridged universal anchor primer. 
gene sequences obtained by the internal sequence amplification, and the upstream (5') and downstream (3') regions were amplified using the rapid amplification of cDNA ends (RACE) method. PCR parameters for the 3' RACE analyses were as follow: initial denaturation at $95^{\circ} \mathrm{C}$ for $2 \mathrm{~min}$, followed by 35 cycles of $95^{\circ} \mathrm{C}$ for $30 \mathrm{~s}, 55^{\circ} \mathrm{C}$ for $1 \mathrm{~min}$, and $72^{\circ} \mathrm{C}$ for $2 \mathrm{~min}$. The $5^{\prime} \mathrm{RACE}$ analyses were performed using kits provided by Invitrogen (Carlsbad, CA), according to the manufacturer's instructions. Internal sequences and PCR products obtained by RACE were electrophoresed in $2 \%$ agarose gel, and DNA was extracted using Quantum Prep ${ }^{\infty}$ Freeze'N Squeeze spin columns (Bio Rad, Hercules, CA) and ligated into the pGEM-T Easy Vector (Promega). Full-length chitinase genes obtained from the liver of $S$. esculenta were amplified using platinum ${ }^{\bullet} P f_{X}$ DNA polymerase (Invitrogen), which has proofreading activity. The reaction conditions were: 35 cycles of $94^{\circ} \mathrm{C}$ for $15 \mathrm{~s}, 55^{\circ} \mathrm{C}$ for $30 \mathrm{~s}$, and $68^{\circ} \mathrm{C}$ for $2 \mathrm{~min}$. The full-length genes obtained were extracted using the same method described for the internal sequence amplification. Base sequences were determined using the Big Dye Terminator Cycle Sequencing FS Ready Reaction Kit (Applied Biosystems, Foster City, CA).

\subsection{Organ Expression of SeChi-1 and SeChi-2}

Total RNA was extracted from $S$. esculenta organs. cDNA was synthesized using $0.5 \mu \mathrm{g}$ of total RNA obtained from each tissue and an oligo dT primer, and amplified using PCR with $1.0 \mu \mathrm{g}$ of the synthesized cDNA, primers for SeChi-1, SeChi-2, and Decembrachiata $\beta$-actin amplification primers (Table 1). The reaction conditions were: 30 cycles of $95^{\circ} \mathrm{C}$ for $30 \mathrm{~s}, 55^{\circ} \mathrm{C}$ for $1 \mathrm{~min}$, and $72^{\circ} \mathrm{C}$ for 2 min.

\subsection{Phylogenetic Analysis of Chitinases}

Phylogenetic analysis, based on the deduced amino acid sequences of the full-length SeChi-1 and SeChi-2 genes, was performed using chitinase genes obtained from multiple organisms. The analysis was performed using ClustalW (http://clustalw.ddbj.nig.ac.jp/) and Tree view.

\section{Results and Discussion}

\subsection{Distribution of Chitinolytic Activities}

Chitinolytic activity measurement using $p \mathrm{NP}-(\mathrm{GlcNAc})_{2}$ and $p \mathrm{NP}-(\mathrm{GlcNAc})_{3}$ as substrates showed that out of eight measured organs of $\mathcal{S}$. esculenta, the liver and stomach, which are involved in digestion, and the posterior salivary gland, which contains chitinase genes that were also found to be present in other cephalopods [33] [34], exhibited high activity (Figure 2(a)). When using glycolchitin as substrate, chitinase activity was detected only liver, stomach, and caecum [37]. Moreover, Hex activity, which is characteristic of exo-type chitinolytic enzymes, was high in the following six organs: the liver, heart, branchial heart, posterior salivary gland, stomach, and caecum (Figure 2(b)). 


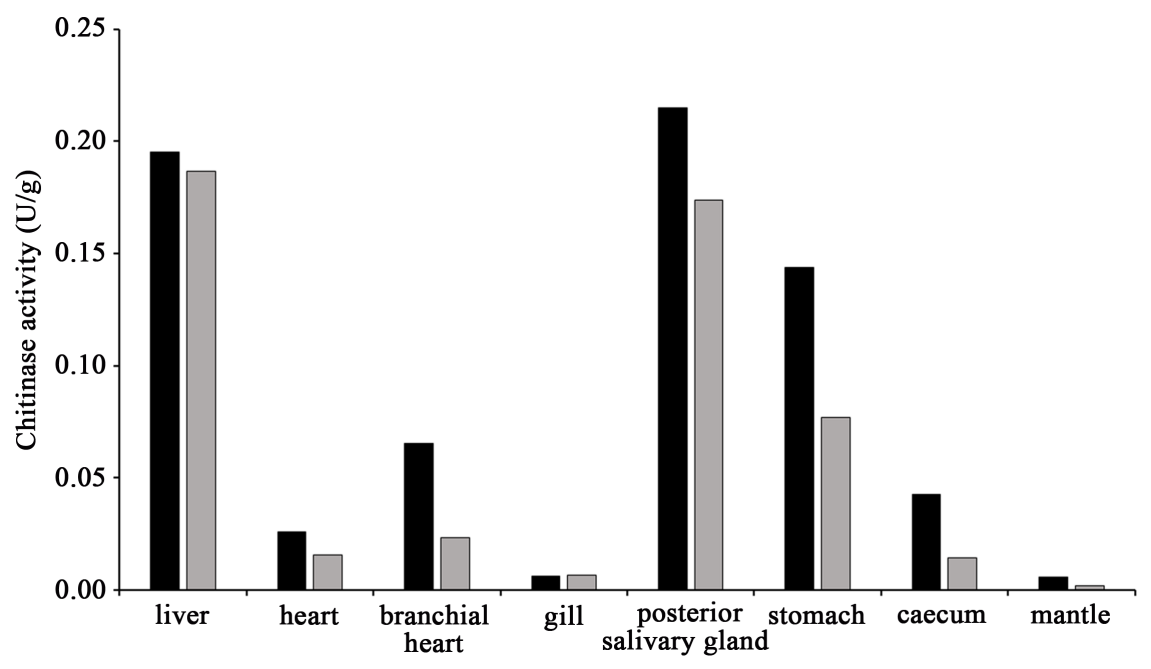

(a)

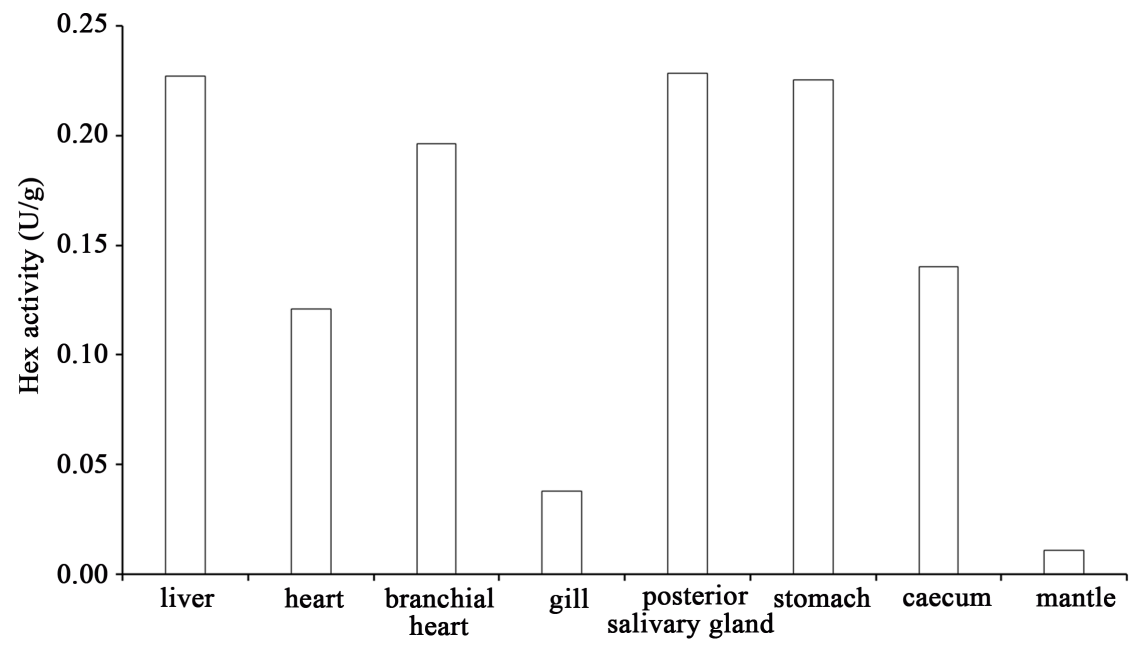

(b)

Figure 2. The distribution of the chitinolytic activities in the organs: (a) Chitinase activity; (b) Hex activity. (-) pNP-(GlcNAc) $)_{2}$; ( ) p pNP-(GlcNAc) ; ( $\square$ ) pNP-(GlcNAc).

In digestive organs, chitinases and Hex are involved in the degradation of chitinous substances following their intake as feed. This is consistent with the feeding habit of $S$. esculenta; that is, $S$. esculenta ingests organisms containing chitinous substances, such as shrimps and crabs [36], suggesting that $S$. esculenta degrades chitin from feed into GlcNAc using both endo- and exo-type enzymes. In addition, it is possible that chitinases in the posterior salivary gland act as a poison, as observed in other cephalopods [33] [34]. Furthermore, because blood chitinases in mollusks have important roles in immunity [23], chitinases in the heart and branchial heart, which are not involved in digestion, are involved in defense against organisms containing chitinous substances, such as parasitic crustaceans and nematodes.

\subsection{Isolation of CBPs from the Liver of S. esculenta}

Using a chitin affinity column, CBPs were separated from the enzyme solution 
obtained from $S$. esculenta liver via $0 \%-70 \%$ ammonium sulfate fractionation (Figure 3). SDS-PAGE was performed using the fraction with the highest chitinolytic activity; CBPs with molecular weights of 52 and $62 \mathrm{kDa}$ (CBP-A, CBP-B) were detected (Figure 4).

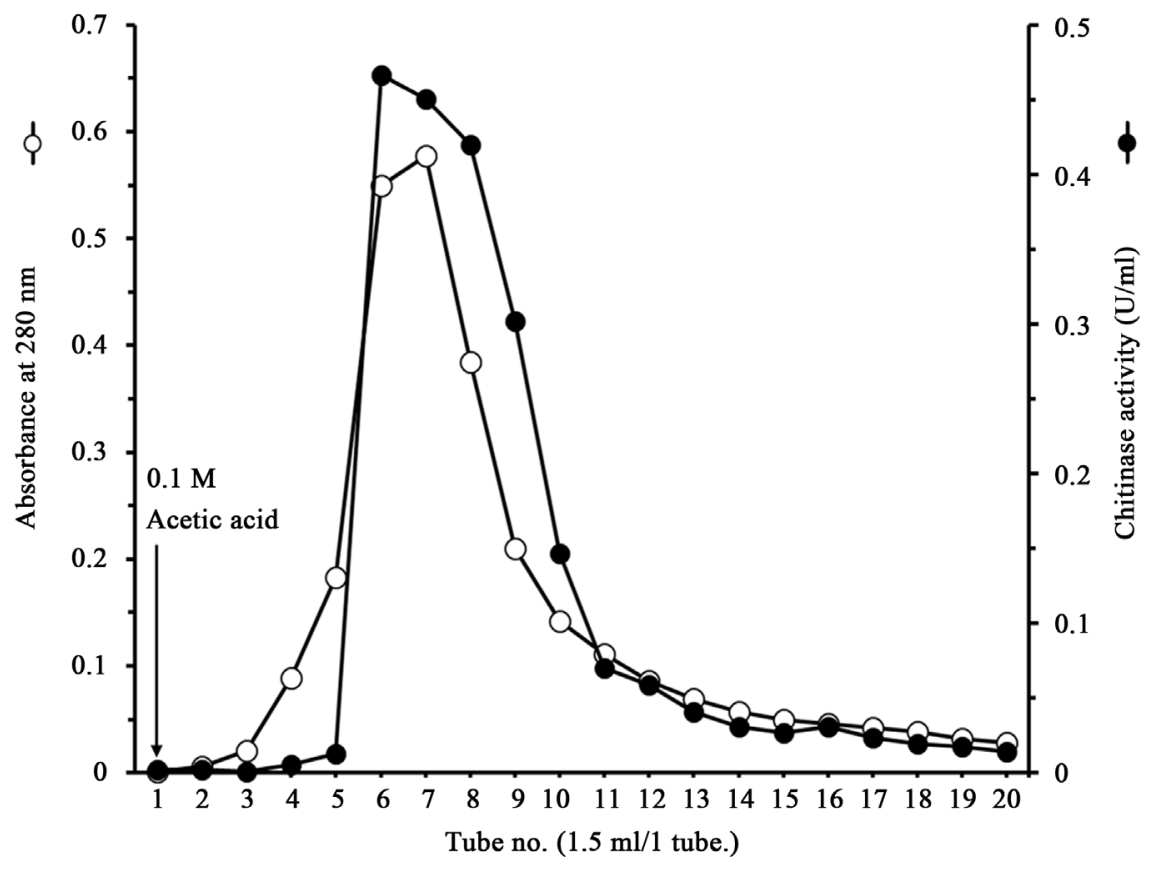

Figure 3. Separation of chitin binding proteins (CBPs) from the liver of $S$. esculenta by using chitin affinity column chromatography. Sample solution was applied to a Chitin EX column previously equilibrated with $20 \mathrm{mM}$ sodium phosphate buffer solution ( $\mathrm{pH}$ 7.2) containing $1 \mathrm{M} \mathrm{NaCl}$, and the non-adsorbed fraction was eluted with the same buffer. The adsorbed fraction was eluted with $0.1 \mathrm{M}$ acetic acid.

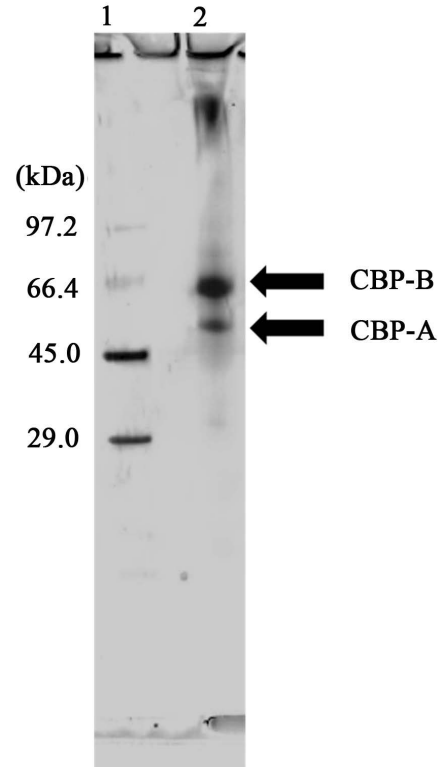

Figure 4. SDS-PAGE of CBPs. (1: Marker; 2: Chitinase active fraction obtained by Chitin EX column chromatography.) 
CBP-B was considered to be SeChi, which is a chitinase purified from the liver of $S$. esculenta [31], because the molecular weight of CBP-B (62 kDa) was consistent with that of SeChi. Two types of chitinase isozymes with molecular weights of 38 [28] and $42 \mathrm{kDa}$ [30] have previously been purified from the liver of Japanese common squid. This suggests that the CBP-A newly detected in this study is an isozyme of chitinase in the liver of $S$. esculenta.

\section{3. cDNA Cloning of Chitinases from the Liver of S. esculenta}

The liver of $S$. esculenta was used as a sample, and internal sequences of chitinase cDNA were amplified by PCR using degenerate primers designed from conserved amino acid sequences of GH family 18 chitinases. As a result, amplified fragments approximately $550 \mathrm{bp}$ in size were found, and two types of base sequences were obtained by base sequencing. NCBI Blast analysis revealed that these base sequences share homology with chitotriosidase of Hawaiian bobtail squid E. scolopes [35]. The upstream and downstream sequences of the aforementioned base sequences were amplified by the RACE method. As a result, initiation and termination codons were identified in the upstream and downstream regions. Then, full-length cDNAs were amplified using Platinum ${ }^{\circledast} P f_{X}$ DNA polymerase.

Two full-length cDNAs, SeChi-1 (1484 bp) and SeChi-2 (1748 bp), were obtained and found to contain 1377-bp (459 amino acids) and 1656-bp (552 amino acids) open reading frames (ORFs), respectively. The molecular weights of SeChi-1 and SeChi-2 were 51.2 and $61.0 \mathrm{kDa}$, respectively, based on the deduced amino acid sequences of $S e C h i-1$ and $S e C h i-2$, (Figure 5 and Figure 6). The molecular weights were very similar to those of CBP-A (52 kDa) and CBP-B (62 $\mathrm{kDa})$, respectively, which were determined by SDS-PAGE. Isoelectric points calculated from the amino acid sequences of SeChi-1 and SeChi-2 were 8.87 and

aAatagtgaattTcCaAgGagatacacattacagcaAa -1

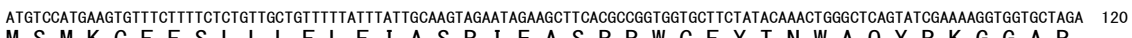
M S M K C F F S L L L L F I A S R I E A S R R W C F Y T N W A Q Y R K G G A R

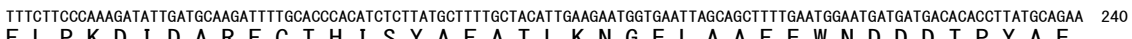
F L P K D I D A R F C T H I S Y A F A T L K N G E L A A F E W N D D D T P Y A E

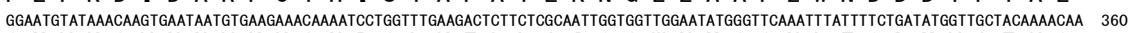

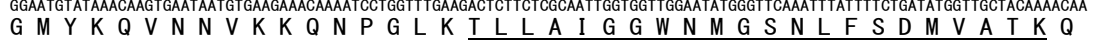

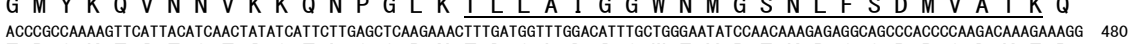

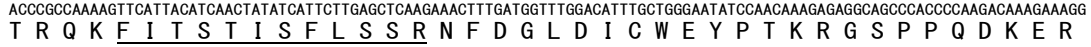

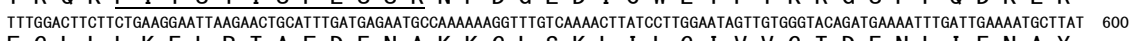
F G L L L K L R T A F D E N A K K GLSKLILGIVVGTDENL I E N A Y

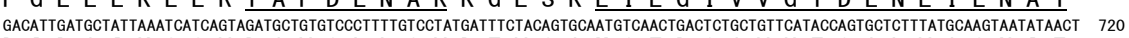
D I D A I K S S V D A V SLLS Y D F Y S A M T D S A V H T S A Y Y A N I T

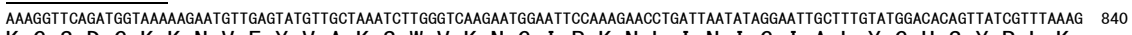
$K G S D G K K N V E Y V A K S W V K N G I P K N L I N I G I A L Y G H S Y R L K$

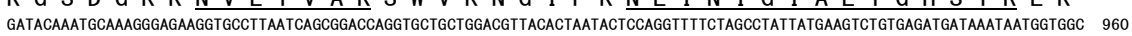

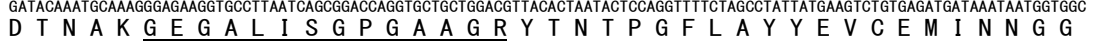

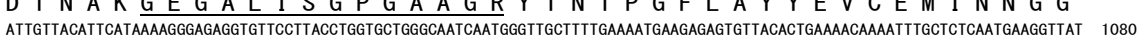

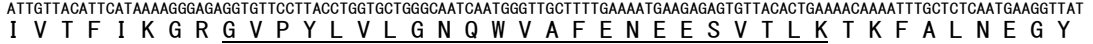

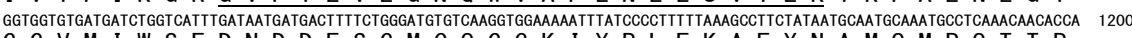
G G V M I W S F D N D F S G M C Q G G K I Y P L F K A F Y N A M Q M P Q T T P

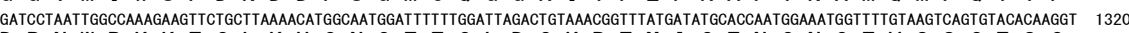
D P N W P K K F L K H G G F F L D C K R F M I C T G N G F V S C T Q G

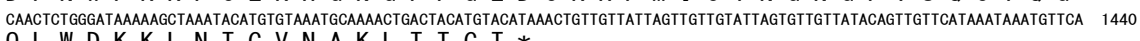
taAattTCaAcaAaAaAaAaAaAaAaAaAa

Figure 5. cDNA and deduced amino acid sequence of SeChi-1. DDBJ accession nos. AB986212. Underlined sequence show matching with the peptide fragments of the separated and trypsinized CBP-A (coverage: 27.23\%, 125 residues). Calculated molecular weight: 51228.59 . Isoelectric point: 8.87 . 


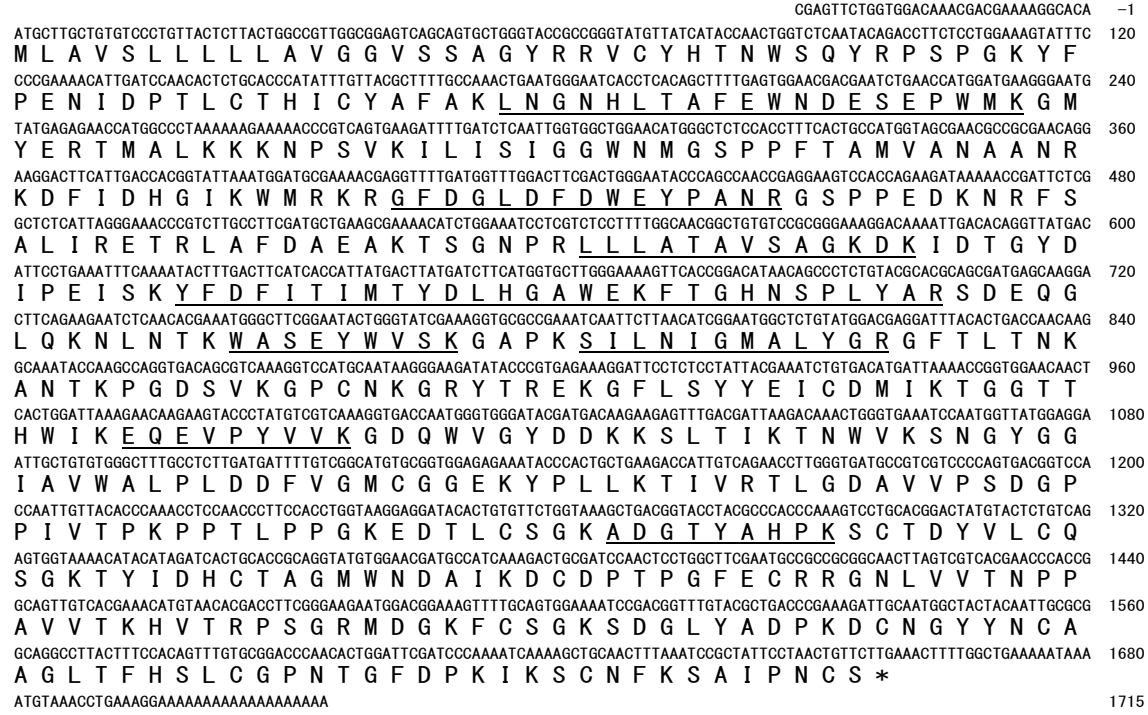

Figure 6. cDNA and deduced amino acid sequence of SeChi-2. DDBJ accession nos.LC319665. Underlined sequence show matching with the peptide fragments of the separated and trypsinized CBP-B (coverage: $21.01 \%, 116$ residues). Calculated molecular weight: 61012.53 . Isoelectric point: 8.79 .

8.79 , respectively. These values are approximate to those of chitinase isozymes purified from the liver of Japanese common squid, at 8.3 [28] and 9.2 [30], suggesting that chitinases from the liver of Decembrachiata are basic proteins.

SeChi-1 and SeChi-2 were found to consist of N-terminal signal peptides, a GH Family 18 catalytic domain, one chitin binding domain (CBD) for SeChi-1, and two CBDs for SeChi-2 (Figure 7). The domain structure of SeChi-2 was consistent with that of a squid chitinase previously reported to possess two CBDs [35]. SeChi-1, which has only one CBD, was revealed to be a new Decembrachiata chitinase.

Figure 8 compares the domain structure of mollusk and fish chitinases. Fish chitinases have previously been reported to contain one CBD [18] [19]. The domain structures of common mackerel stomach chitinases (SjChi-1 and SjChi-2) are shown in Figure 8 as examples. Conversely, the domain structures of mollusk chitinases are diverse; a chitinase from the gonad of sea hare $(A k C h i)$ and a chitinase from Japanese oyster chitinase 3 ( $C g C h i-3)$ possess no CBD; $S$. esculenta liver chitinase 1 (SeChi-1) and a snail Biomphalaria glabrata chitinase 3-like protein 1 (BgChi-3lp1) possess one CBD; and S. esculenta liver chitinase 2 (SeChi-2) and chitinase 3 from the mantle of Hyriopsis cumingii (HcChi-3) possess two CBDs. This suggests that mollusk chitinases have several domain structure, which correspond to their physiological roles.

\subsection{Amino Acid Sequence of the Chitinases}

Two CBPs (CBP-A and CBP-B) (Figure 3 and Figure 4) obtained from the liver of $S$. esculenta were fragmented into peptides by trypsin treatment and compared with the amino acid sequences of SeChi- 1 and SeChi-2 via proteome analysis. 


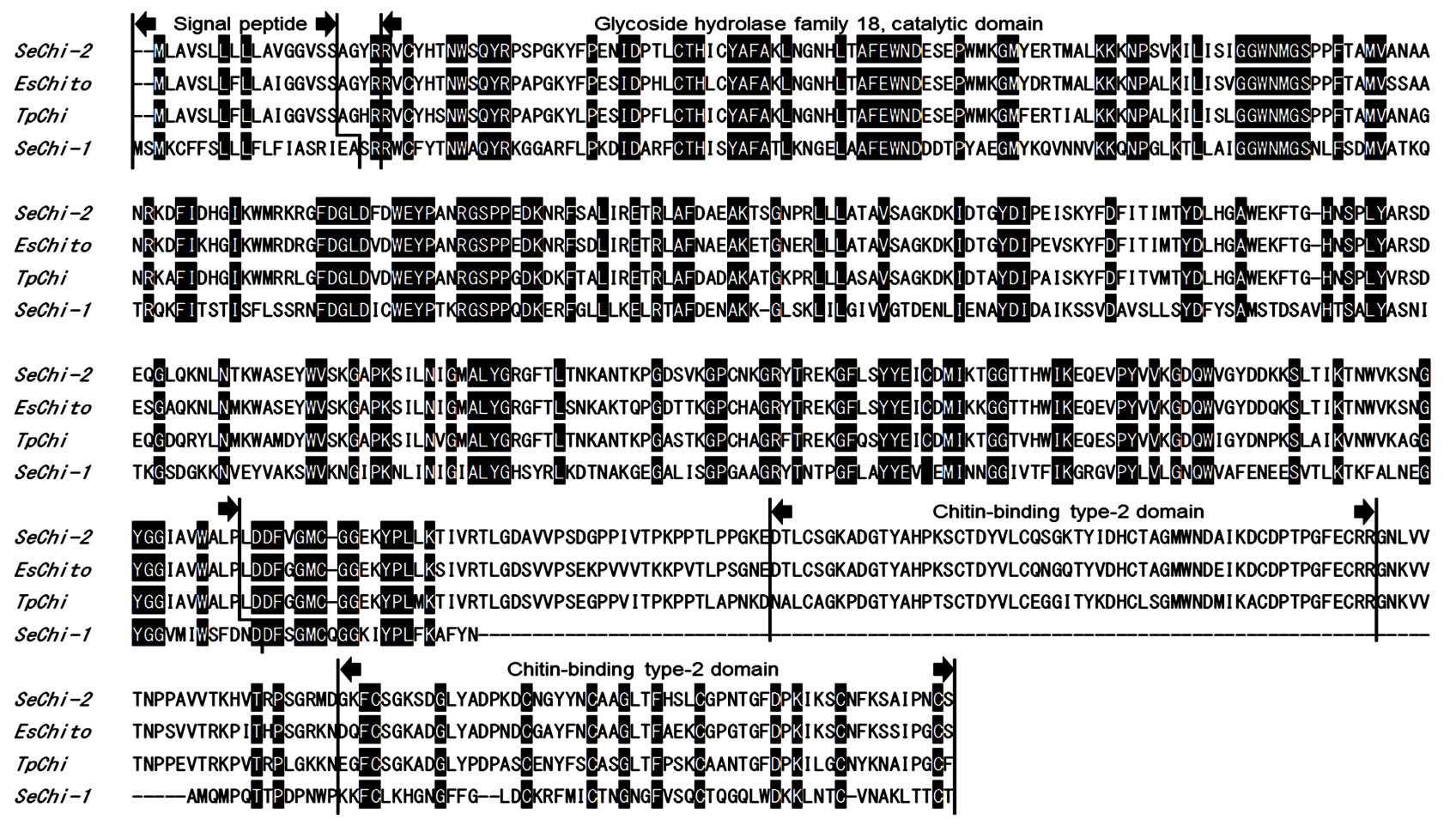

Figure 7. Multiple alignment of deduced amino acid sequences of $S$. esculenta Chitinases (SeChi-1 and SeChi-2) with Euprymna scolopes chitotriosidase (EsChito), and Todarodes pacificus Chitinase (TpChi). GenBank accession nos.: EsChito, AHM92582.1; TpChi, LC146770. Matched sequences are shown in black.

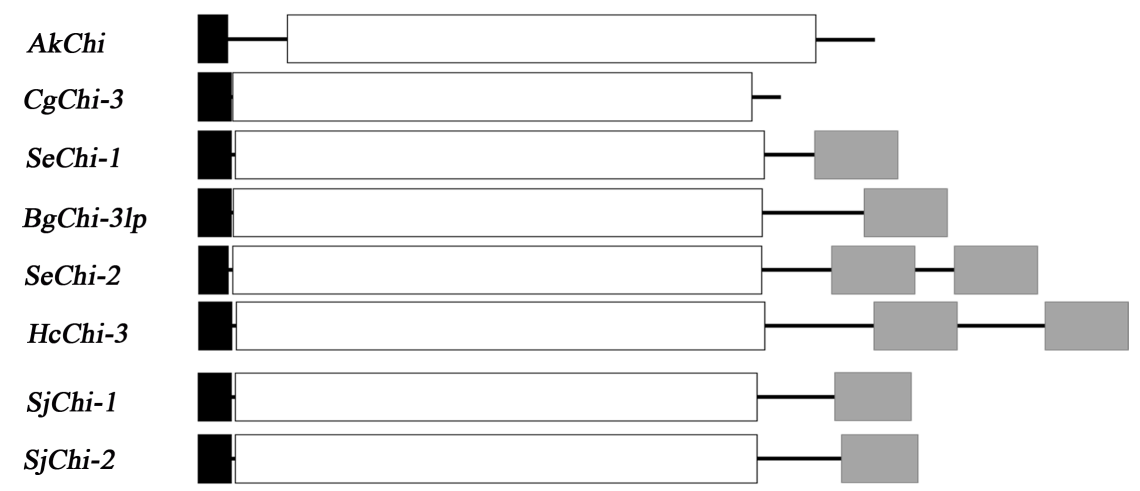

Figure 8. The schematic representation molluscan and fish chitinases. Black boxes show signal peptide. White boxes show GH family 18 catalytic domain. Gray boxes show the chitin-binding type-2 domain. GenBank accession nos.: AkChi, BAS44269; CgChi-3, AJ971239; BgChi-3Ip, XP013090777; HcChi-3, AFO53261; SjChi-1, AB686657; SjChi-2, AB689022.

A sequence obtained from peptide fragments of CBP-A was consistent with the amino acid sequence of SeChi-1 (coverage: 27.23\%, 125 residues) (Figure 5). A sequence obtained from the peptide fragments of CBP-B was consistent with the amino acid sequence of SeChi-2 (coverage: $21.01 \%, 116$ residues) (Figure 6). These results indicate that SeChi-1 and SeChi-2 encode CBP-A and CBP-B, respectively. In other words, CBP-A was a protein band of chitinase isozyme SeChi-1 and CBP-B was a protein band of chitinase isozyme SeChi-2. 
Trypsin is reported to cleave the C-terminal side of lysine $(\mathrm{K})$ and arginine $(\mathrm{R})$ [39]. It was confirmed that trypsin worked adequately at all cleavage sites because all of the obtained peptides ended with $\mathrm{K}$ or $\mathrm{R}$.

\subsection{Organ Expression of SeChi-1 and SeChi-2}

The expression of SeChi-1 and SeChi-2 was investigated in eight S. esculenta organs, with both genes found to be expressed only in the liver (Figure 9). Expression of SeChi-2 was stronger than that of SeChi-1 (Figure 9). This result was consistent with findings from SDS-PAGE of CBPs isolated from the liver, such that the CBP-B band encoded by SeChi-2 was thicker than that of CBP-A encoded by SeChi-1 (Figure 4). Because SeChi-1 and SeChi-2 were found to be expressed in the liver, where chitinolytic activity was found, SeChi-1 and $S e$ Chi-2 are suggested to encode enzymes involved in chitin degradation in this organ. Furthermore, although high chitinolytic activity was detected in the posterior salivary gland, as shown in Figure 2, neither SeChi-1 nor SeChi-2 were expressed there. The presence of chitinases acting as poison in the posterior salivary gland of other cephalopods has been reported [33] [34]. Additionally, the presence of chitinases isozymes, which differ from SeChi-1 and SeChi-2, is suggested in the posterior salivary glandof $S$. esculenta.

\subsection{Phylogenetic Analysis of SeChi-1 and SeChi-2}

On the basis of amino acid sequence homology, phylogenetic analysis was conducted using SeChi-1, SeChi-2, GH family 18 chitinases of other organisms, and a GH family 18 chitinase of Serratiamarcescens, as an outgroup. SeChi-2 formed a group with other Decembrachiata chitinases, whereas SeChi-1 did not form a group with any of the mollusk chitinases (Figure 10). Considering that SeChi-1 is the first chitinase with one CBD to be identified in Decembrachiata (Figure 8), it was considered to be a new-type chitinase.

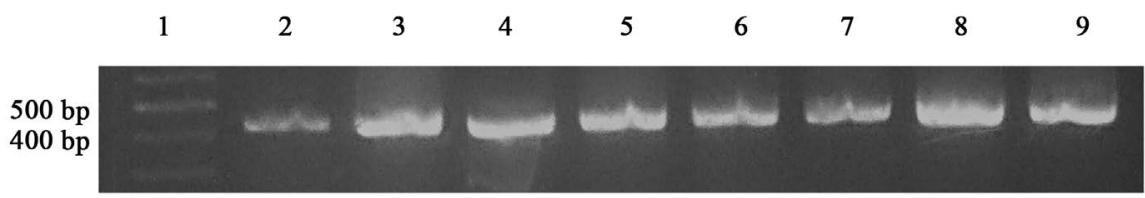

(a)

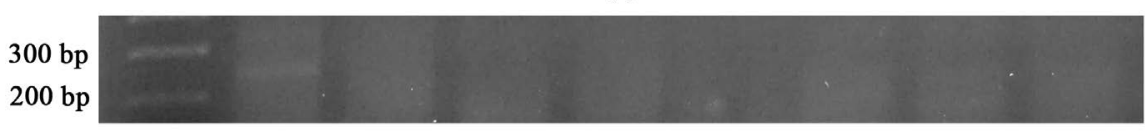

(b)

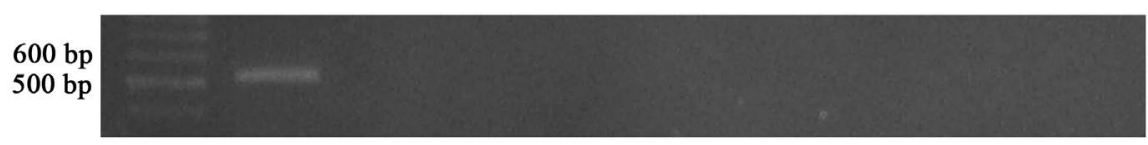

(c)

Figure 9. Chitinase and $\beta$-actin expressions in various organs. (a) $\beta$-actin; (b) $\operatorname{SeChi} 1$; (c) SeChi-2. (M, markers; 1 , liver; 2 , heart; 3 , branchial heart; 4 , gill; 5 , posterior salivary gland; 6, stomach; 7, caecum; 8 , mantle). 


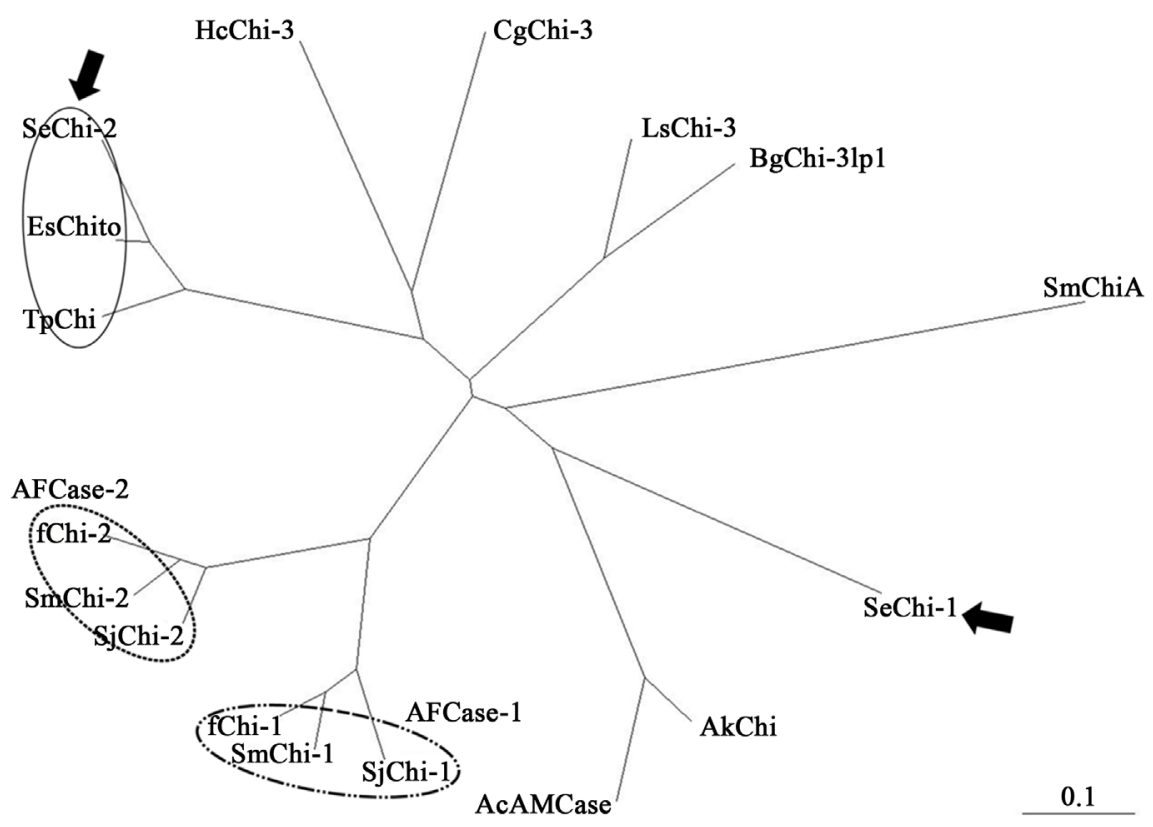

\begin{tabular}{|c|c|c|}
\hline Abbreviation & Species & Accession number \\
\hline SeChi-1 & Sepia esculenta & AB986212 \\
\hline SeChi-2 & Sepia esculenta & LC319665 \\
\hline TpChi & Todarodes pacificus & LC146770 \\
\hline EsChito & Euprymna scolopes & KF015222 \\
\hline HcChi-3 & Hyriopsis cumingii & JN582038 \\
\hline CgChi-3 & Crassostrea gigas & AJ971239 \\
\hline AcAMCase & Aplysia californica & XM005112601 \\
\hline AkChi & Aplysia kurodai & LC085435 \\
\hline BgChi-3lp1 & Biomphalaria glabrata & XP013090777 \\
\hline LsChi-3 & Lymnaea stagnalis & LC069028 \\
\hline SjChi-1 & Scomber japonicus & BAL40979 \\
\hline SjChi-2 & Scomber japonicus & BAL41779 \\
\hline SmChi-1 & Sebastiscus marmoratus & AB686658 \\
\hline SmChi-2 & Sebastiscus marmoratus & AB686659 \\
\hline fChi-1 & Paralichthys olivaceus & AB121732 \\
\hline fChi-2 & Paralichthys olivaceus & AB121733 \\
\hline SmChiA & Serratia marcescens & X03657 \\
\hline
\end{tabular}

Figure 10. Phylogenetic analysis of chitinase amino acid sequences by the neighbor-joining method of the program ClustalW. A bacterial chitinase, Serratia marcescens chitinase, was used as the out group. The scale bar indicates the substitution rate per residue. The arrows show SeChi-1 and SeChi-2 obtained in the present study.

\section{Conclusion}

In this study, the distribution of chitinolytic enzyme activity in $S$. esculenta was measured and high chitinolytic activity was found in digestion-related organs such as the liver. These chitinases can potentially degrade ingested chitinous substances. In addition, high chitinolytic activity was observed in the posterior 
salivary gland. Chitinases in the posterior salivary gland may act as a poison, as observed in other cephalopods. Chitinolytic activity in other organs suggests that chitinases are involved in defense against parasites and other activities. Two CBPs (CBP-A and CBP-B) with molecular weights of 52 and $62 \mathrm{kDa}$, respectively, were separated from the liver of $S$. esculenta. The molecular weight of CBP-B was consistent with that of SeChi, a chitinase previously purified from the liver of $S$. esculenta. CBP-A was suggested to be a chitinase isozyme obtained from the liver of $S$. esculenta. Full-length cDNAs (SeChi-1, SeChi-2) encoding two chitinases (SeChi-1, SeChi-2) were obtained from the liver of $S$. esculenta. The molecular weights of SeChi-1 and SeChi-2 calculated from their amino acid sequences were 51.2 and $61.0 \mathrm{kDa}$, respectively, and their isoelectric points were 8.87 and 8.79 , respectively, indicating that they are basic proteins. SeChi-1 contained one CBD and SeChi-2 contained two CBDs. Peptide fragments of CBPs isolated from the liver of $S$. esculenta were analyzed and compared with the amino acid sequences of SeChi-1 and SeChi-2 by proteome analysis. A sequence obtained from peptide fragments of CBP-A was consistent with the amino acid sequence of SeChi-1 (27.23\%) and a sequence obtained from peptide fragments of CBP-B was consistent with the amino acid sequence of SeChi-2 (21.01\%). Among the $S$. esculenta organs studied, SeChi-1 and SeChi-2 were only expressed in the liver. This suggests that the two chitinases are involved in chitin degradation in the liver. The two chitinase genes were not expressed in the posterior salivary gland, where high chitinolytic activity was detected. This suggests that other chitinase isozymes are present in the posterior salivary gland. Phylogenetic analysis revealed that SeChi-2 formed a group with other Decembrachiata chitinases, whereas SeChi-1 did not group with mollusk chitinases. Considering that SeChi-1 represents the first chitinase to possess one CBD in Decembrachiata, SeChi-1 is considered to be a new-type chitinase.

\section{Acknowledgements}

This work was supported in part by College of Bioresource science, Nihon University Grant (2017).

\section{References}

[1] Arbia, W., Arbia, L., Adour, L. and Amrane, A. (2013) Chitin Extraction from Crustacean Shells Using Biological Methods-A Review. Food Technology and Biotechnology, 51, 12-25.

[2] Karthik, N., Akanksha, K., Binod, P. and Pandey, A. (2014) Production, Purification and Properties of Fungal Chitinases-A Review. Indian Journal of Experimental Biology, 52, 1025-1035.

[3] Komatsu, M., Son, J., Matsushita, N. and Hogetsu, T. (2007) Fluorescein-Labeled Wheat Germ Agglutinin Stains the Pine Wood Nematode, Bursaphelenchusxyiophilus. Journal of Forest Research, 13, 132-136. https://doi.org/10.1007/s10310-008-0065-9

[4] Khoushab, F. and Yamabhai, M. (2010) Chitin Research Revisited. Marine Drugs, 8, 1988-2012. https://doi.org/10.3390/md8071988 
[5] Patil, R.S., Ghormade, V.V. and Deshpande, M.V. (2000) Chitinolytic Enzymes: An Exploration. Enzyme and Microbia Technology, 26, 473-483. https://doi.org/10.1016/S0141-0229(00)00134-4

[6] Slámová, K., Bojarová, P., Petrásková, L. and Kren, V. (2010) $\beta$ - $N$-acetylhexosaminidase: What's in a Name...? Biotechnology Advances, 28, 682-693. https://doi.org/10.1016/j.biotechadv.2010.04.004

[7] Gooday, G.W. (1999) Aggressive and Defensive Roles for Chitinases. Cellular and Molecular Life Sciences, 87, 157-169.

[8] Henrissat, B. and Barroch, A. (1993) New Families in the Classification of Glycosyl Hydrolases Based on Amino Acid Sequence Similarities. Biochemical Journal, 293, 781-788. https://doi.org/10.1042/bj2930781

[9] Fukamizo, T., Koga, D. and Goto, S. (1995) Comparative Biochemistry of Chitinases-Anomeric Form of the Reaction Products. Bioscience Biotechnology Biochemistry, 59, 311-313. https://doi.org/10.1271/bbb.59.311

[10] Koga, D., Yoshioka, K. and Arakane, Y. (1998) HPLC analysis of Anomeric Formation and Cleavage Pattern by Chitinolytic Enzyme. Bioscience Biotechnology Biochemistry, 62, 1643-1646. https://doi.org/10.1271/bbb.62.1643

[11] Shinya, S., Nagata, T., Ohnuma, T., Taira, T., Nishimura, S. and Fukamizo, T. (2012) Backbone Chemical Shifts Assignments, Secondary Structure, and Ligand Binding of a Family GH-19 Chitinase from Moss, Bryumcoronatum. Biomolecular NMR Assignments, 2, 157-161. https://doi.org/10.1007/s12104-011-9346-X

[12] Ikeda, M., Miyauchi, K., Mochizuki, A. and Matsumiya, M. (2009) Purification and Characterization of Chitinase from the Stomach of Silver Croaker Pennahiaargentatus. Protein Expression and Purification, 65, 214-222.

[13] Ikeda, M., Miyauchi, K. and Matsumiya, M. (2012) Purification and Characterization of a $56 \mathrm{kDa}$ Chitinase Isozyme (PaChiB) from the Stomach of Silver Croakerpennahiaargentatus. Bioscience Biotechnology Biochemistry, 76, 971-979. https://doi.org/10.1271/bbb.110989

[14] Kurokawa, T., Uji, S. and Suzuki, T. (2004) Molecular Cloning of Multiple Chitinase Genes in Japanese Flounder, Paralichthysolivaceus. Comparative Biochemistry and Physiology B, 138, 255-264.

[15] Ikeda, M., Kondo, Y. and Matsumiya, M. (2013) Purification, Characterization, and Molecular Cloning of Chitinases from the Stomach of the Threeline Grunt Parapristipomatrilineatum. Process Biochemistry, 48, 1324-1334.

[16] Kakizaki, H., Ikeda, M., Fukushima, H. and Matsumiya, M. (2015) Distribution of Chitinolytic Enzymes in the Organs and cDNA Cloning of Chitinase Isozymes from the Stomach of Two Species of Fish, Chub Mackerel (Scomber japonicus) and Silver Croaker (Pennahiaargentata). Open Journal of Marine Science, 5, 398-411. https://doi.org/10.4236/ojms.2015.54032

[17] Kawashima, S., Ikehata, H., Tada, C., Ogino, T., Kakizaki, H., Ikeda, M., Fukushima, H. and Matsumiya, M. (2016) Stomach Chitinase from Japanese Sardine Sardinopsmelanostictus: Purification, Characterization, and Molecular Cloning of Chitinase Isozymes with a Long Linker. Marine Drugs, 14, 1-13. https://doi.org/10.3390/md14010022

[18] Matsumiya, M. Kakizaki, H. and Ikeda, M. (2017) Prosperity Strategy and Chitinase in Fish. Chitin and Chitosan Research, 23, 4-16.

[19] Ikeda, M., Kakizaki, H. and Matsumiya, M. (2017) Biochemistry of Fish Stomach Chitinase. International Journal of Biological Macromolecules, 104, 1672-1681. 
[20] Wang, G.L., Xu, B., Bai, Z.Y. and Li, J.L. (2012) Two Chitin Metabolic Enzyme Genes from Hyriopsiscumingii: Cloning, Characterization, and Potential Functions. Genetics and Molecular Research, 11, 4539-4551. https://doi.org/10.4238/2012.October.15.4

[21] Yonezawa, M., Sakuda, S., Yoshimura, E. and Suzuki, M. (2016) Molecular Cloning and Functional Analysis of Chitinases in the Fresh Water Snail, Lymnaeastagnalis. Journal of Structural Biology, 196, 107-118.

[22] Li, H., Wang, D., Denq, Z., Huang, G., Fan, S., Zhou, D., Liu, B., Zhang, B. and Yu, D. (2017) Molecular Characterization and Expression Analysis of Chitinase from the Pearl Oyster Pinctadafucata. Comparative Biochemistry and Physiology Part B: Biochemistry \& Molecular Biology, 203, 141-148.

[23] Badariotti, F., Lelong, C., Dubos, M.P. and Favrel, P. (2007) Characterization of Chitinase-Like Proteins (Cg-Clp1 and Cg-Clp2) Involved in Immune Defence of the Mollusk Crassostreagigas. The FEBS Journal, 274, 3646-3654. https://doi.org/10.1111/j.1742-4658.2007.05898.x

[24] Badariotti, F., Thuau, R., Lelong, C., Dubos, M.P. and Favrel, P. (2007) Characterization of an Atypical Family 18 Chitinase from the Oyster Crassostreagigas. Evidence for a Role in Early Development and Immunity. Developmental \& Comparative Immunology, 31, 559-570.

[25] Gao, L., Xu, G. J., Su, H., Gao, X.G., Li, Y.F., Bao, X.B., Liu, W.D. and He, C.B. (2014) Identification and Expression Analysis of cDNA Encoding Chitinase-Like Protein (CLP) Gene in Japanese Scallop Mizuhopectenyessoensis. Genetics and Molecular Research, 13, 10727-10740. https://doi.org/10.4238/2014.December.18.14

[26] Matsunaga, G., Karasuda, S., Nishino, R., Fukushima, H. and Matsumiya, M. (2016) Molecular Cloning of a Chitinase Gene from the Ovotestis of Kuroda's Sea Hare Aplysiakurodai. Advances in Bioscience and Biotechnology, 7, 38-46. https://doi.org/10.4236/abb.2016.71005

[27] Yang, B., Zhang, M., Li, L., Pu, F., You, W. and Ke, C. (2015) Molecular Analysis of Atypical Family 18 Chitinase from Fujian Oyster Crassostreaangulata and Its Physiological Role in the Digestive System. PLoS ONE, 10, e0129261. https://doi.org/10.1371/journal.pone.0129261

[28] Matsumiya, M. and Mochizuki, A. (1997) Purification and Characterization of Chitinase from the Liver of Japanese Common Squid Todarodespacificus. Fisheries Science, 63, 409-413. https://doi.org/10.2331/fishsci.63.409

[29] Matsumiya, M., Miyauchi, K. and Mochizuki, A. (2002) Characterization of 38kDa and $42 \mathrm{kDa}$ Chitinase Isozymes from the Liver of Japanese Common Squid Todarodespacificus. Fisheries Science, 68, 603-609. https://doi.org/10.1046/j.1444-2906.2002.00467.x

[30] Matsumiya, M., Miyauchi, K. and Mochizuki, A. (2003) Purification and Some Properties of a Chitinase Isozyme from the Liver of Japanese Common Squid Todarodespacificus. Fisheries Science, 69, 427-429. https://doi.org/10.1046/j.1444-2906.2003.00640.x

[31] Nishino, R., Suyama, A., Ikeda, M., Kakizaki, H. and Matsumiya, M. (2014) Purification and Characterization of a Liver Chitinase from Golden Cuttlefish, Sepia esculenta. Journal of Chitin and Chitosan Science, 2, 238-243. https://doi.org/10.1166/jcc.2014.1065

[32] Kondo, H., Morita, T., Ikeda, M., Kurosaka, C., Shitara, A., Honda, Y., Nozaki, R., Aoki, T. and Hirono, I. (2010) Identification of Enzyme Genes in the Liver of the Bleeker's Squid Loligobleekeri by Expressed Sequence Tag Analysis. Fisheries 
Science, 76, 161-165. https://doi.org/10.1007/s12562-009-0182-1

[33] Fry, B.G., Roelants, K. and Norman, J.A. (2009) Tentacles of Venom: Toxic Protein Convergence in the Kingdom Animalia. Journal of Molecular Evolution, 68, 311-321. https://doi.org/10.1007/s00239-009-9223-8

[34] Ruder, T., Sunagar, K., Undheim, E.A., Ali, S.A., Wai, T.C., Low, D.H., Jackson, T.N., King, G.F., Antunes, A. and Fry, B.G. (2013) Molecular Phylogeny and Evolution of the Proteins Encoded by Coleoid (Cuttlefish, Octopus, Squid) Posterior Venom Glands. Journal of Molecular Evolution, 76, 192-204.

https://doi.org/10.1007/s00239-013-9552-5

[35] Kremer, N., Philipp, E.E., Carpentier, M.C., Brennan, C.A., Kraemer, L., Altura, M.A., Augustin, R., Häsler, R., Heath-Heckman, E.A., Peyer, S.M., Schwartzman, J., Rader, B.A., Ruby, E.G., Rosenstiel, P. and McFall-Ngai, M.J. (2013) Initial Symbiont Contact Orchestrates Host-Organ-Wide Transcriptional Changes that Prime Tissue Colonization. Cell Host \& Microbe, 14, 183-194.

[36] Alves, D.M., Cristo, M., Sendao, J. and Borges, T.C. (2006) Diet of the Cuttlefish Sepia officinalis (Cephalopoda: Seplidae) off the South Coast of Portugal (Eastern Algarve). Journal of the Marine Biological Association of the United Kingdom, 86, 429-436. https://doi.org/10.1017/S0025315406013312

[37] Matsumiya, M., Miyauchi, K. and Mochizuki, A. (1998) Distribution of Chitinase and $\beta$-N-Acetylhexosaminidase in the Organs of a Few Squid and a Cuttlefish. Fisheries Science, 64, 166-167. https://doi.org/10.2331/fishsci.64.166

[38] Ohtakara, A. (1988) Chitinase and $\beta$ - $N$-acetylhexosaminidase from Pycnoporuscinnabarinus. Method in Enzymology, 161, 462-470.

[39] Olsen, J.V., Ong, S.E. and Mann, M. (2004) Trypsin Cleaves Exclusively C-Terminal to Arginine and Lysine Residues. Molecular \& Cellular Proteomics, 3, 608-614. https://doi.org/10.1074/mcp.T400003-MCP200 